\title{
Generic Framework for Context-Aware Communication Services in Visitor's Guides
}

\author{
Tsvi Kuflik ${ }^{1}$, Pnina Soffer ${ }^{1}$, Iris Reinhartz-Berger ${ }^{1}$, Sadek Jbara ${ }^{1}$, Oliviero Stock ${ }^{2}$ \\ ${ }^{1}$ Department of Information Systems, University of Haifa, Haifa, Israel; ${ }^{2}$ FBK-irst, Trento, Italy. \\ Email: \{tsvikak, spnina, iris\}@is.haifa.ac.il, sjbara@gmail.com, stock@fbk.eu \\ Received April 2 ${ }^{\text {nd }}, 2011$; revised April 12 ${ }^{\text {th }}, 2011$; accepted April 21 ${ }^{\text {st }}, 2011$.
}

\begin{abstract}
Ubiquitous computing plays an increasing role in our lives. Typically, applications in ubiquitous computing environments are context aware, namely, they react to the situations of their users at a given moment in time. One example for such environment is visitor's guides in cultural heritage sites, supporting visits of individuals or small groups, such as families or friends. In such environments, it is well known that interaction among visitors enhances the overall visit experience. Recently, some research prototypes of visitor's guides have started supporting such interaction through textual communication services embedded in them. However, these applications have so far been developed separately in an ad-hoc manner, despite common features and infrastructures they share. The research described here generalizes communication services offered by different visitor's guides and suggests a systematic and generic framework for developing context-aware communication services for visitor's guides. The specific communication services are abstracted into a domain model, later used in practice for adapting and tailoring the different concepts to the specific requirements of the applications. The framework is demonstrated in the specific setting of a multi-agent museum visitor's guide system. We also show that the suggested framework is not limited to the specific museum visitor's guide system but may facilitate the development of context-aware communication applications in general.
\end{abstract}

Keywords: Communication Services, Context Aware Communication, Visitor's Guides, Domain Analysis

\section{Introduction}

In the past decade, when mobile technology has matured enough, many research prototypes of context aware mobile tour guides and museum visitor's guides appeared, aimed at providing museum visitors with personalized and context aware information. Most of the works focused on exploring how the novel technology can be applied for supporting individuals, mainly by providing context aware information and navigation support to visitors in museums [1-6]. In many cases, these systems supported also interaction among visitors by providing them with some communication services. For example, Rantanen et al. [7] suggested enabling users to "post" virtual messages for each other's attention at specific physical locations.

Interaction among museum visitors is known to enhance the visit experience [8]. Moreover, since a group visit is a common form in museums [9-11] and other cultural heritage sites, the focus of the research now turns to supporting groups in these places $[12,13]$. In such scenarios, technology-supported communication between museums visitors enables them to interact even when they are not in close proximity. As such, communication services should be considered not as a minor add-on, but as a central group-supporting service in the applications. Context awareness of such services makes them an integral part of the visit experience for groups.

To the best of our knowledge, all the works done so far in the area of context aware communication services have developed each service separately in an ad-hoc manner. This makes the development of each service a considerable effort, and limits the variety of services that can be offered by a specific system. We claim that these services can be considered a domain that exhibits a large variety of features, fulfilling different needs, but with common features that characterize all or most services in the domain. Since all these communication services share common basic characteristics, and possibly a common infrastructure, it would be reasonable to utilize the commonalities and all the possible variations and provide a basis over which specific services can be easily developed. An easy development process will facilitate the inclusion of 
a variety of communication services in Visitor's Guide systems. Following this idea, this paper addresses context aware communication services in a systemic way, through domain analysis. While there is an abundance of research about context awareness in general, we focus on the com-munication services themselves. To this end, a generic framework for defining and implementing context aware communication services has been developed, providing a configurable infrastructure, which can be tailored to meet the various needs and requirements of specific communication services. The framework has been implemented and demonstrated in an "active museum" environment, which is one example of a visitor's guide. The environment is composed of a physical space containing exhibits and computation equipment (communication infrastructure, computers, presentation devices, etc.). Specifically, the framework was applied within a multi-agent museum visitor's guide system, developed as a part of the PIL ${ }^{1}$ project, an Italian-Israeli research collaboration project dealing with Personal Experience with Active Cultural Heritage.

The rest of the paper is structured as follows. Section 2 provides the background for the current research and reviews related work. Section 3 introduces the suggested framework for defining context-aware communication services, developed through analyzing the domain of interest. Section 4 reports an implementation of the framework in a museum visitor's guide system, whereas Section 5 evaluates this framework through application case studies. Conclusions and future research directions are given in Section 6.

\section{Background and Related Work}

This section provides background about context-aware services and applications in general, and communication services in visitor's guides in particular. It motivates our work on context aware communication services and is a basis for generalization.

\subsection{Context Aware Services and Applications}

Defining "Context” is a challenging task. Many researches in the area of context awareness tried to define the context of a user in a way suited to their application needs. Dey et al. [14] claim that "while most people tacitly understand what context is, they find it hard to elucidate". They defined context as "any information that can be used to characterize the situation of an entity", whereas an entity is defined as "a person, place, or object that is considered relevant to the interaction between a user and an application, including the user and applications themselves". This definition of context can be spe-

${ }^{1}$ http://www.cri.haifa.ac.il/connections/pil/ cialized when dealing with a specific application or a family of applications. For example, Roffia [15] specialized context definition to mobile cultural heritage applications, stating that “context is a coordinate's pair, named physical and logical coordinates. The physical coordinate represents the current user's position and orientation. The logical coordinate represents information on the level of detail explicitly provided by the user."

A context-aware service is a service which uses context-related information and adapts itself to the changes of user's and environment's context dynamically and automatically. A large number of context aware applications have been proposed under the vision of ubiquitous computing in general and visitor's guides in particular. An early example of con-text-aware computing is the Olivetti Active Badge system [16]. Based on emitted infrared signals from a badge, the system determined users location information. The most common usage of the system was by a receptionist who routinely used it while forwarding telephone calls from the main switchboard. The receptionist would look at the display of locations and then redirect the telephone call to the correct location.

In the cultural heritage domain, the "Guide" project [17] provided an electronic handheld guide that enabled visitors to Lancaster access city information, create tailored city tours, and access interactive services, such as making ticket reservations or booking hotel accommodations. It also included communication services among visitors, reviewed later on in this paper.

Context aware systems can also use physiological sensors like in StartleCam [18], developed at MIT media lab. This system is composed of a wearable video camera, a computer, and a sensing system, enabling the camera to be controlled via both conscious and preconscious events involving the wearer. A skin conductivity signal is measured, related to the attention level. Traditionally, a wearer consciously hits record on the video camera, or runs a computer script to trigger the camera according to some prespecified frequency. The system offers an additional option: images are saved by the system when it detects certain events of supposed interest to the wearer.

Developing context aware application is not easy and it draws a lot of research attention in recent years, as demonstrated by numerous conferences and workshops addressing this issue. Many attempts were made to provide generic infrastructure for general context aware application development (to list just a few, see [14,19-24], some even for the specific setting of cultural heritage sites [25,26]. However, most of them focused on context modeling and infrastructure, rather than on the services themselves. Conversely, we focus on communication services, assuming that an application can use one of the 
existing context modeling tools.

\subsection{Communication Services in Visitor's Guides}

Context aware communication services have been included in various systems and especially in mobile context aware museums and city guides. Since such services are the focus of interest in our work, we surveyed 55 mobile tourist guide systems developed from the early 1990s until recently, only with respect to these services. Twelve of them provide textual communication services, which are of particular relevance to museums, where voice-based communication might not be welcomed. These systems are presented and discussed below in detail (grouped by type, in chronological order). They serve as a basis for analyzing and defining the domain of interest.

\subsubsection{City Guides}

The Campiello system $[27,28]$ aims to encourage the creation of connected communities in cultural towns. Using paper-based interfaces, Campiello enables the visitors to add information to a personal diary. These pages can be printed or faxed in order to be processed later. Furthermore, people can post their paper forms to large screens, and they can immediately view the newest input to the system together with others, to get immediate feedback on their input.

Smart Sight [29] is a voice activated tourist guide system. It provides its visitors with "Tourist Diary" to help them organize their trip experience. When the tourist arrives at a point of interest, the system logs the position and time retrieved from the GPS and system clock. The tourist can ask the system to take a picture or digital video clip, and to add captions to the picture or dictate the description or comments during his/her visit at the point of interest. After the tourist finishes visiting the site or the day ends, he/she can ask the system to generate an HTML document based on the stored information.

GUIDE $[17,30]$ an electronic handheld tour guide already introduced in Section 2.1, provides its users with personalized location aware information. It also enables visitors to send and receive messages to/from companions, sharing their experiences with others.

LoL@ [31] is a mobile electronic tour guide designed for tourists visiting the city center of Vienna. It provides predefined tours through the city center, information about the sights, navigation and routing, and an electronic tour diary. All visited places are automatically included in a tour diary stored on the application provider's server. Additionally, users can include comments and private data like digital photographs. The diary can be retrieved after returning from the trip and can be accessed by people staying at home during the trip. As fu- ture work the authors suggest to consider immediate messages to be sent between visitors during their tour (real time tour reporting).

\subsubsection{Museum Guides}

CyberGuide [1,32] is a mobile, context aware tour guide that allows its users to send messages to exhibit owners and to report their location to a central server, accessible by others.

Hippie [33] is a nomadic museum guide developed within the HIPS (Hyper-Interaction within the Physical Space) project [34]. It allows its users to take notes and annotate visited exhibits in order to store personal explanations or bookmarks available during the visit. It further supports sending immediate messages that can be directed to a dedicated addressee, such as family or group members in a museum, or to a specified full e-mail address, to contact a remote user.

Exploratorium Guidebook [35], a location aware visitor's guide developed for the Exploratorium in San Francisco $^{2}$, provides two communication functionalities, named "rememberer" and "communicator". The first one provides the visitors with means to build a record of their experiences which they can consult during and after their visit, while the second one helps visitors communicate by electronic bulletin boards for individual exhibits, instantmessaging, and/or beaming information between handheld devices. The researchers conclude that their remembering service may have value for personal and social uses. They also conclude that people seem to enjoy helping each other and discussing the exhibits, and this seems to encourage additional interaction with the exhibits.

MUSE [36], a location aware museum guide, allows visitors to mark the currently displayed screen or to use a built-in camera to take a picture of what they are looking at and update a "visit memory album". At any time, either during or at the end of the visit, the visitors can modify the memory album by deleting some selected items, re-ordering them, or including comments and annotations. The final album can be saved on CDs, which represent the "memory" of the on-site visits.

Virtual Heritage [37] is the focus of a research that explores the possibility of using virtual reality in order to enrich visitors experience on sites. The system also allows visitors to take notes and exchange information with peers in a chat-like mechanism.

MOMO [38] allows visitors to communicate and send messages to other visitors, and to see the names of visitors that already visited a specific artwork, in order to share similar interests at the museum or to keep in touch more easily. The researchers claim that the messaging feature is the main point of the social interaction because

\footnotetext{
http://www.exploratorium.edu/
} 
it allows visitors to communicate and to interact with the rest of the museum visitors, either by sending messages individually or by sending the same message to all the members of a group.

The exSite museum visitor's guide, made by ESPRO ${ }^{3}$, offers MyCollection, a service that allows its users to mark artworks of interest and select later on objects to be printed at the museum shop.

\subsubsection{Messaging Services}

InfoRadar [7] provides public and private location-based messaging and a novel radar interface for accessing messages, where nearby messages can be tracked in the mobile device on a map. Such messages have an expiry time and visibility, and can be viewed remotely if the recipient is unlikely to visit the location of the message. The InfoRadar package contains a compact-size digital camera that can be connected via infrared to the InfoRadar main device and allows the users to attach a picture to a message being composed.

\subsubsection{Summary of Communication Services Support in Visitor's Guides}

In summary, after reviewing 55 mobile visitor's guides, we found that about $20 \%$ of them (i.e., 12 systems) provide text-based communication services to their users. Table 1 presents the classification of these systems according to the types of communication services they provide and the contextual aspects they consider: an immediate message sent to a recipiant, a virtual "Post-It" left at a location (e.g., personalized Geo-Note), or a "memory" -message to self (e.g., personal diary entry). The prevailing contextual aspects found in the cultural heritage guides involve Spatial, Temporal and Social aspects, whereas the contextualized information is an adaptation of the information delivered to the visitors based on contextual aspects.

As can be seen, the various applications, which represent a variety of communications services suggested by Visitor's Guides, share some common features, but each has its uniqueness too. Looking at the services, most of them can be categorized either as immediate messages or diary-like applications and some applications provide more than one type of communication services. As far as contextual aspects, most of them are location aware, where about half provide contextualized information. Most of them also have some form of social awareness (that is tightly coupled with communication services). In the following section we use all this information in order to get a generalized view of the domain under consideration.

${ }^{3}$ http://www.espro.com/
Table 1. Classification of the reviewed systems according to communication service types* and type of context.

\begin{tabular}{|c|c|c|c|c|c|c|c|c|}
\hline & \multirow{2}{*}{ System } & \multicolumn{3}{|c|}{$\begin{array}{l}\text { Comm. } \\
\text { service* }\end{array}$} & \multicolumn{4}{|c|}{ Type of Context } \\
\hline & & $\mathbf{I}$ & $\mathbf{P}$ & D & $\begin{array}{l}\text { Cont } \\
\text { Info. }\end{array}$ & Spatial & Temp. & Soc. \\
\hline \multirow{4}{*}{ 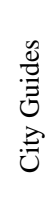 } & Campiello $^{\#}$ & $\mathrm{X}$ & & $\mathrm{X}$ & & & & $\mathrm{X}$ \\
\hline & Smart Sight & & & $\mathrm{X}$ & $\mathrm{X}$ & $\mathrm{X}$ & $\mathrm{X}$ & \\
\hline & GUIDE & $\mathrm{X}$ & & & & $\mathrm{X}$ & & $\mathrm{X}$ \\
\hline & LoL@ & $\mathrm{X}$ & & $\mathrm{X}$ & & $\mathrm{X}$ & & $\mathrm{X}$ \\
\hline \multirow{7}{*}{ 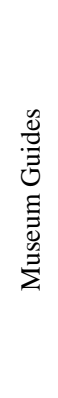 } & CyberGuide & $\mathrm{X}$ & & & & $\mathrm{X}$ & & $\mathrm{X}$ \\
\hline & HIPPIE & $\mathrm{X}$ & & $\mathrm{X}$ & $\mathrm{X}$ & $\mathrm{X}$ & & $\mathrm{X}$ \\
\hline & $\begin{array}{l}\text { Exploratorium } \\
\text { GuideBook }\end{array}$ & $\mathrm{X}$ & & $\mathrm{X}$ & $\mathrm{X}$ & $\mathrm{X}$ & & $\mathrm{X}$ \\
\hline & MUSE & & & $\mathrm{X}$ & $\mathrm{X}$ & $\mathrm{X}$ & & \\
\hline & Virtual Heritage & & & $\mathrm{X}$ & & & & \\
\hline & MOMO & $\mathrm{X}$ & & & & & & $\mathrm{X}$ \\
\hline & exSite & & & $\mathrm{X}$ & $\mathrm{X}$ & & & \\
\hline $\begin{array}{l}\text { Msg. } \\
\text { serv. }\end{array}$ & InfoRadar & & $\mathrm{X}$ & & & $\mathrm{X}$ & $\mathrm{X}$ & $\mathrm{X}$ \\
\hline
\end{tabular}

*Immediate (I), Post it (P) or Diary (D); "The type of context in the service provided by Campiello is not specific, and depends on the user's choice

\section{A Generic Framework for Developing Context Aware Communication Services}

As noted, the variety of systems is quite large. However, some guidelines constraining their common features can be outlined. First, each communication service has one sender and potentially several recipients, one of which may be the sender (e.g., in diary communication services). In most cases, the sender and recipient are all users or visitors using the system to be developed and they are usually identified by IPs and/or names. The communication service itself is characterized by its name, subject, sending time, and the content to be sent.

Each user (or visitor) may have different context attributes in various time periods. As already noted, these context attributes can be divided into four categories:

1) Contextualized Information includes general attributes regarding the visiting environment that the user (visitor) is exposed to, including physical objects and presentations. In active museums, the physical objects may be exhibits, and the presentations may be audio or video files about exhibits and exhibitions.

2) Spatial Context includes the current location and movement of the visitor. The spatial context is characterized by the area, the specific location (e.g., a specific exhibition in an active museum), direction (of movement), orientation (indicating where the visitor focus is 
and possibly the general view, and speed.

3) Temporal Context includes attributes such as current time, visit phase, phase start time, and visit duration.

4) Social Context refers to groups of users (visitors) and to the particular role of a user in the group, as well as aspects such as attitude and mood.

Even if we do not use explicitly the term "cognitive context" it is clear we mean (and other systems may also implicitly aim) to take into account the physical and the perceptive, epistemic and possibly emotional state of the visitor. We have chosen this level of representation to accommodate various experiences; and also for our own work (see the following) we prefer not to be bound to a specific model, but just to propose the generic elements that can then be used at a higher level to provide a characterization of the cognitive context.

Note that the context of a particular visitor changes over time during the visit. Furthermore, potentially, a user can change groups during the visit. Thus, context aware communication services should be able to refer to the current and past context of their users, both senders and recipients. Examples of involving contextual aspects of the sender include adding to a communication service the location of the sender or information regarding his/ her group. Considering the context of a recipient, messages, for instance, are not sent to a recipient who has already visited the exhibit or left the museum. For supporting these, each context aware communication service is connected to different context objects, representing conditions on the sender, the recipients, and the environment. These context objects may be embedded in the service as contextual conditions and/or contextualized content. Contextual conditions define constraints on the delivery of a given message. They usually use at least the context of the recipient. Contextualized content, on the other hand, usually refers to the sender's context to be included in the message.

Contextual conditions can be further divided into present and future contextual conditions. Evaluation of pre- sent contextual conditions at run time determines whether the message should be sent or not. They are evaluated once at delivery time. Future contextual conditions, on the other hand, should be dynamically evaluated with every change in contextualized data. Messages that have future contextual conditions will be delivered as soon as their (future) contextual conditions are satisfied. Regarding the types of messages defined above, immediate messages have no future contextual conditions, whereas postit services must have such conditions. Note that diary services are characterized by the equality between their sender and recipient. Table 2 exemplifies how to specify context aware communication services in the suggested framework, whereas Figure 1 depicts the domain model of the suggested framework, expressed as a class diagram.

Note that in general the domain model includes concepts that are not specific to visitor's guides. The only part that is specific to visitor's guides is the specialized context (contextualized information, spatial context, temporal context, and social context). In addition, the specialized context aware communication services in the model (i.e., immediate, post-it, and diary) rely on our analysis, which was done for that area. Hence, this list may not be complete when considering other application areas.

\section{Implementation of the Generic Framework in Active Museums}

As seen in the domain model, there exists a solid common basis to the various communication services that can be offered in visitor's guides. We propose to utilize this understanding of the commonality and variability to enable easy development of a variety of services that may be required by different applications. These services will be provided by configuring a common and generic communication service infrastructure. This way, when specific applications are developed, specific communication services can be rapidly provided as configurations of the generic service. Such architecture can streamline the development process of new applications that require

Table 2. Context aware communication services in the suggested framework.

\begin{tabular}{|c|c|c|c|c|}
\hline Communication Service & $\mathbf{T}^{*}$ & present condition $^{\#}$ & future condition & content \\
\hline $\begin{array}{l}\text { Send a message to all the users in the sender's group } \\
\text { who have not visited the area in which the sender is } \\
\text { visiting now, calling them to visit this area }\end{array}$ & I & $\begin{array}{l}\text { Group (recipient) = Group (sender) } \\
\text { AND NOT Area (sender) in } \\
\text { VisitHistory. Area (recipient) }\end{array}$ & & Area (sender) \\
\hline $\begin{array}{l}\text { Send a message to all the users in the sender's group } \\
\text { who have not visited the exhibit the sender is visiting } \\
\text { now to watch this exhibit as soon as they enter its } \\
\text { area, but only if this happens in the next } 5 \text { minutes }\end{array}$ & $\mathrm{P}$ & $\begin{array}{l}\text { Group (recipient) = Group(sender) } \\
\text { AND NOT Exhibit (sender) in } \\
\text { VisitHistory. Exhibit (recipient) }\end{array}$ & $\begin{array}{l}\text { Area }(\text { recipient) }=\text { Area } \\
\text { (sender) AND Current } \\
\text { Time (recipient) } \leq \\
\text { Message.SentTime }+5\end{array}$ & Exhibit (sender) \\
\hline $\begin{array}{l}\text { Remind the sender regarding the area in which he/she } \\
\text { is visiting now }\end{array}$ & $\mathrm{D}$ & sender = recipient & & Area (sender) \\
\hline
\end{tabular}

*Immediate (I), Post it (P) or Diary (D); ${ }^{*}$ VisitHistory refers to the past context of a user (sender or recipient). 


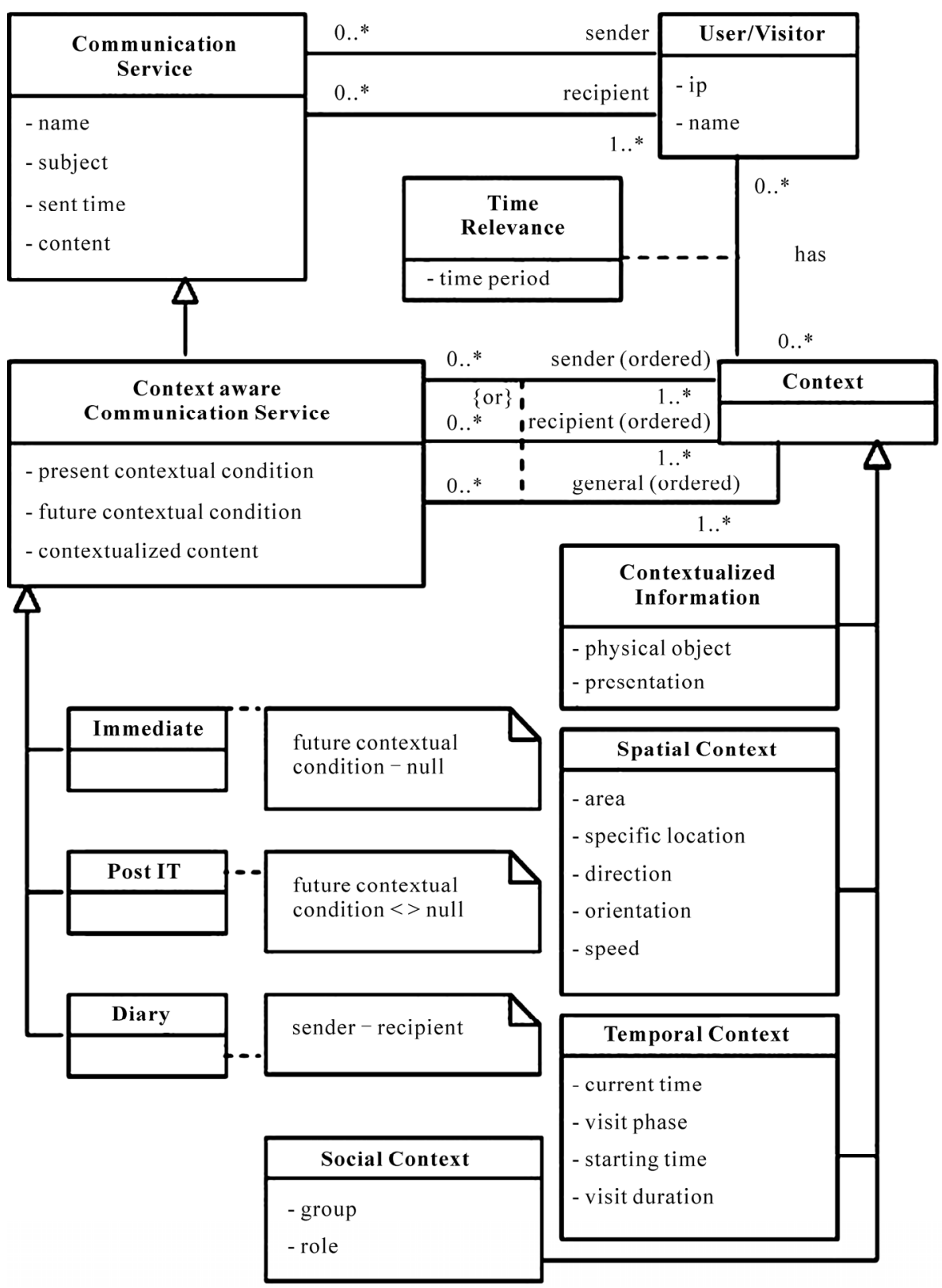

Figure 1. A domain model of context aware communication services in visitor's guides.

context aware communication services.

Considering museum visitor's guides as a wide and interesting sub-area of visitor's guides, we have developed a tool that allows its users (application developers) to define and implement communication services to be used as part of their applications automatically. The specific communication services are specialized classes, defined as configurations of the generic context aware communication service class from Figure 1. These service classes are defined using a Service Configuration tool which yields XML files, specifying the exact configuration of the communication services at hand (including their con- textual conditions and their contextual content). The XML definition files are then used as inputs by a Communication Generator tool that generates the specific services for the used communication infrastructure. Nevertheless, the user interface and the actual logic of each generated communication service have to be developed individually to meet the particular requirements of the service. In our case, the services themselves are generated as agents in a multi-agent system that has been developed as part of the PIL project [13], a multi-agent museum visitor's guide system. This system uses LoudVoice agents' communication infrastructure [39,40] and 
contains five components (see Figure 2): 1) a Spatial Information Broker that reports the visitor's position periodically, integrating positioning information from different sources (currently Infrared and WiFi); 2) a Presentation Composer that provides presentations to the visitor, based on the current location and taking into account the visitors' inferred preferences; 3) a User Modeler agent that keeps track of the visitors' preferences based on their behavior; 4) a Communication Services Agent that provides communication services to the visitors; and 5) a User Assistant (Device Interface) agent which is installed on the user's PDA and provides the user interface to the system (information presentation/user actions).

For specific service definitions, the "communication services generator" interprets the XML communication definitions and generates the required communication services as specialized agents. Note that the generic service idea is not limited to a specific infrastructure. It can be implemented as a web service, over a multi-agent communication infrastructure, or through other communication mechanisms. In general, it can be implemented over any communication infrastructure that supports the definition of the generic model, as will be discussed later to show the generality of the framework.

The communication service development and usage process includes four consequential parts, participating in different life-cycle stages and depicted in Figure 3:

1) At design time, the communication services, as required by the application, are defined using the Communication Service Definition tool, producing an XML communication services definition file.

2) At application development time, specific application that includes the code for using the communication activities (usually user interface that will use the com- munication service) is developed. It includes requests for sending or receiving services as defined by the XML configuration file (at design time).

3) At runtime initialization stage, the communication services are automatically created by the communication service generator, based on the XML-based configuration file produced at design time.

4) After initialization of runtime, active users utilize the communication services through the user interfaces. Any operation, either sending or reading a specific message is invoked by a user activating the appropriate application that was developed for this specific communication service. The communication itself as well as the collection and interpretation of contextual information required for the service are provided by the generic infrastructure.

\section{Demonstration and Evaluation of the Generic Framework}

This section demonstrates the usage of our generic framework in four communication services: SMS (i.e., immediate messages), Post-It, Memories (i.e., diary), and Visit Reminder (also diary) services. Then, we evaluate the generality of the framework by showing how it can be applied to produce the services reviewed in Section 2 .

\subsection{Demonstration: SMS and Post It-Like Services}

We start with presenting the implementation of two services which have already been integrated into the PIL system, namely the SMS and Post it-like services. While SMS is a relatively simple immediate message service, the virtual post-it service is more complicated, with a major role to context awareness. The implementation in-

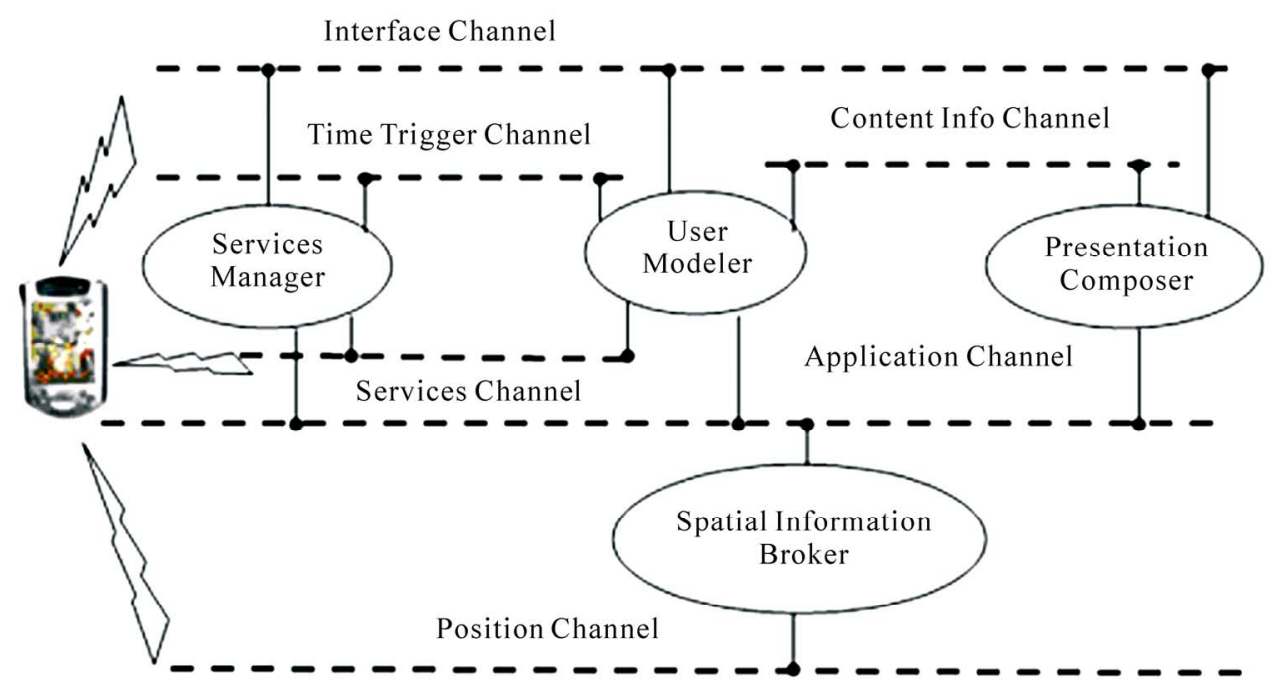

Figure 2. The museum visitor's guide architecture in PIL. 


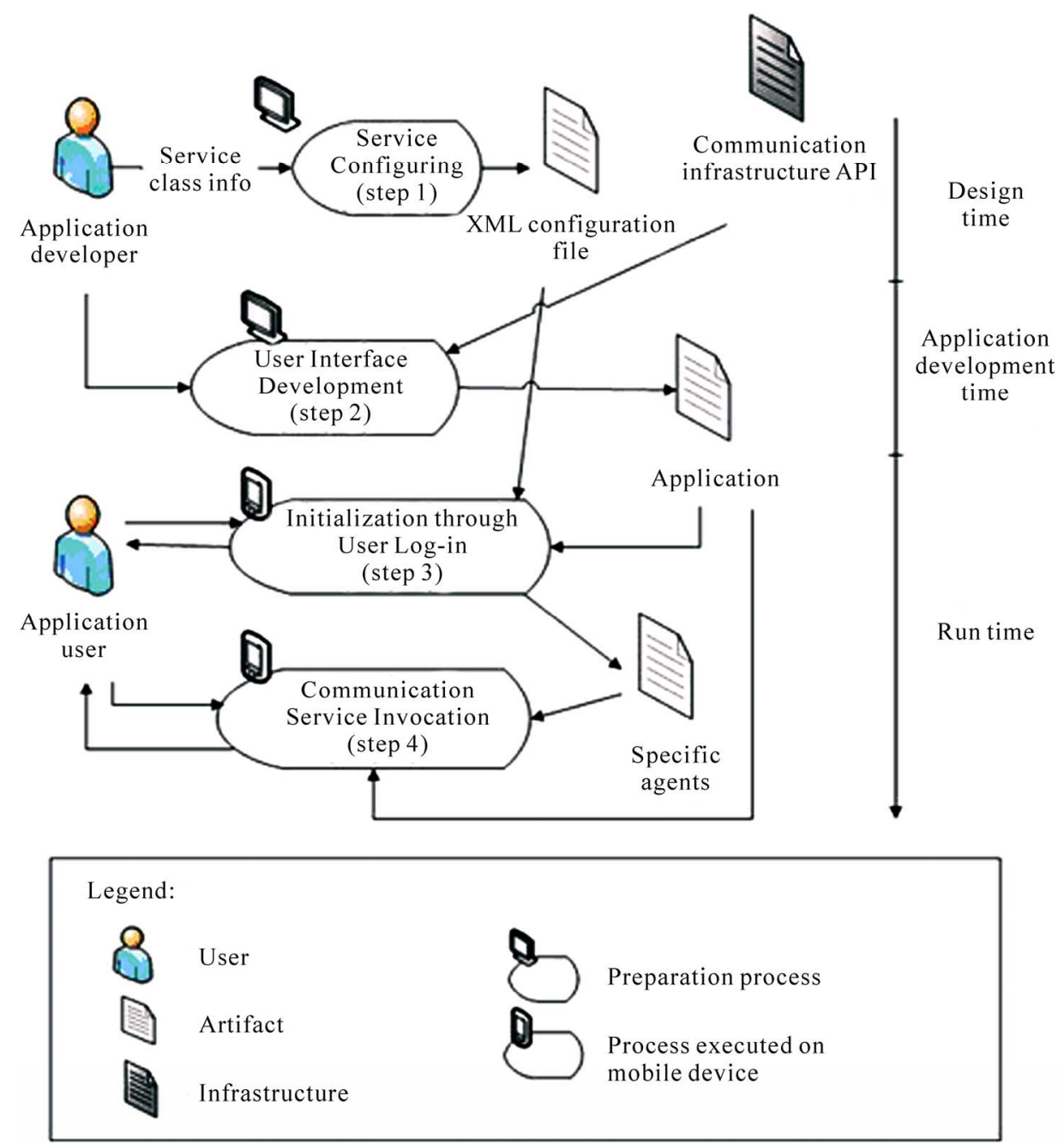

Figure 3. The suggested communication service development and usage process.

cluded the possibility to select and send a message from a set of predefined messages. This is important in this case since museum visitors should focus their attention on the exhibits and not on the guide itself.

\subsubsection{Requirements and Usage Scenarios}

The SMS-like messages can be classified as immediate messages. The requirements from this type of services are that messages should be delivered immediately after sending, taking into account the current context of the sender and optionally of the recipient. In the PIL project, when the user is in a certain position, next to a specific exhibit and wants to send a message to a friend, the user selects a message from a list of predefined ones, including: 1) "You should see this exhibit", 2) "You should visit this area", 3) "You should skip this area", and 4) "Could you please come here?" (Without giving any specific details) If, for example, the sender selects the message "you should see this exhibit", the message will be delivered to a recipient if he/she has not yet visited the sender's current exhibit, and the information about "this exhibit", like its name and an image, will be added automatically by the system to the delivered message. This information may possibly include a path on a map that leads the recipient to the sender's location as well (since the two positions, this of the sending user and this of the recipient are known to the system).

Like SMS, Post-It services are context-based virtual notes whose delivery depends on the context of the sender and recipient. Unlike SMS, the post-it note may be virtually attached to an exhibit that the sender is looking at, and the message will be delivered to the recipients only when and if they visit this exhibit (and optionally if additional contextual conditions are fulfilled). Post-It services may take into account the visit history of the recipient, the current context of the sender, and the social context of both sender and recipient. The content of a delivered message is enhanced by the sender's current 
contextual information. As an example to a Post-It note consider: "Remember this exhibit-I'd like to talk to you about it later". The message will be delivered to the recipient if he/she arrives at the sender's (current) location within a given time period (the message expiry time), and if he/she has not yet visited the sender's current exhibit. Furthermore, information about "this exhibit" will be added to the message. This information will suggest the recipient to choose the exhibit recommended by the sender when (and if) visiting the location where the postit is attached.

\subsubsection{Configuration Process}

In order to define these two services, fulfilling the above requirements, the developer of an application configures a generic communication service, using a configuration tool developed for this purpose The result of the process is an XML definition file, where each communication service is defined as a class element with a unique identifier and two sub-elements, conditions and content. Figure 4 represents the SMS definition file.

The conditions element includes information on present and future conditions to handle the current communication service, as well as possibility to initializing variable values needed for defining contextual content or conditions. The SMS configuration file (Figure 4) includes only present conditions, specifying that the recipient should be in the sender's group and has not visited the area the sender is currently visiting. The Post-It configuration file includes both present and future conditions. The present conditions require that the recipient is in the sender's group and has not visited the exhibit the sender is currently visiting; future conditions specify that the message has to be delivered to a recipient that arrives at the area the sender is currently in within 5 minutes.

The content element includes the contextual element in the actual content of the communication service. The SMS service should augment the area of the sender (name, picture) into the message, while the Post-It message should include the exhibit information (current exhibit of the sender). The XML file is used during system initialization to generate the relevant communication services, as described below.

\subsubsection{Runtime Application}

In addition to the configuration definition file, an appropriate user interface for these services has been developed. For the SMS service, the application is executed in runtime based on the user's selection of a predefined message (listed at the beginning of Section 5.1.1, and on the second screen from left in Figure 5).

At initialization time, i.e., when the system is initialized, communication service agents are created following the definitions in the configuration file for every logged-in user. At runtime, while the visitors are using the guide, they can press the button of the envelope (the icon $\square$ in the left bottom of Figure 5) in order to open a list of predefined text messages (Figure 5, second screen from the left). After the sender chooses the desired message from the list ("you should see this exhibit" in this case), a list of other visitors' names appears (Figure 5, third screen from the left) to enable the sender to choose the actual recipients (one or all). A final screen includes the text message and recipient names to confirm sending (Figure 5, rightmost screen). In the example the sender recommends the recipient to see the exhibit at which she is currently looking.

Upon delivery, a button at the bottom of the screen notifies a recipient that a message is received (the icon in Figure 6, left screen). If he/she chooses to read the message by pressing the button, then a list of sender names appears (Figure 6, second screen from the left), and when one is selected, a list of messages sent by that visitor is presented (Figure 6, third screen from the left). Finally, after choosing one of the messages, a new screen with the delivered message appears. Recall that the content of the delivered message depends on the sender's context. Specifically, the phrase "this exhibit" is replaced with the name of the exhibit that was recommended by the sender, and an image of this exhibit is added to the message (Figure 6, rightmost screen).

Runtime application of the Post-It service is similar to that of the SMS, with the following differences. First, the list of possible messages is slightly different (see Figure 7 left side). In the given list, messages 4 and 5 match the configuration specified in Figure 5, where the message is attached to an Area and bearing the information of an Exhibit in that area as its content. In contrast, the first three messages $(1,2$, and 3 ) relate to a slightly different configuration. These messages are supposed to be attached to an Exhibit rather than to an Area, and their content provides a recommendation about some Presentations rather than Exhibits. This would mean different

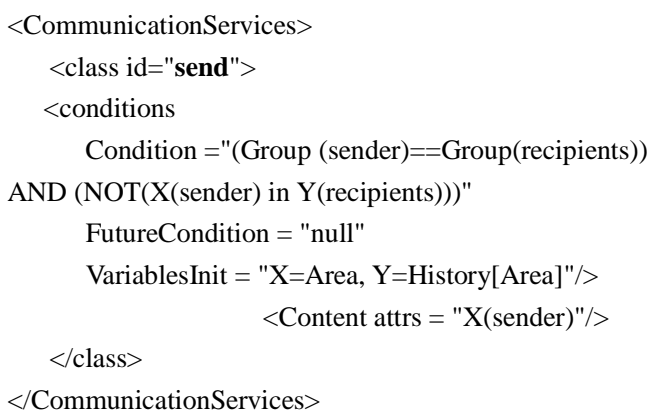

Figure 4. The XML configuration file for the SMS-like immediate message service. 

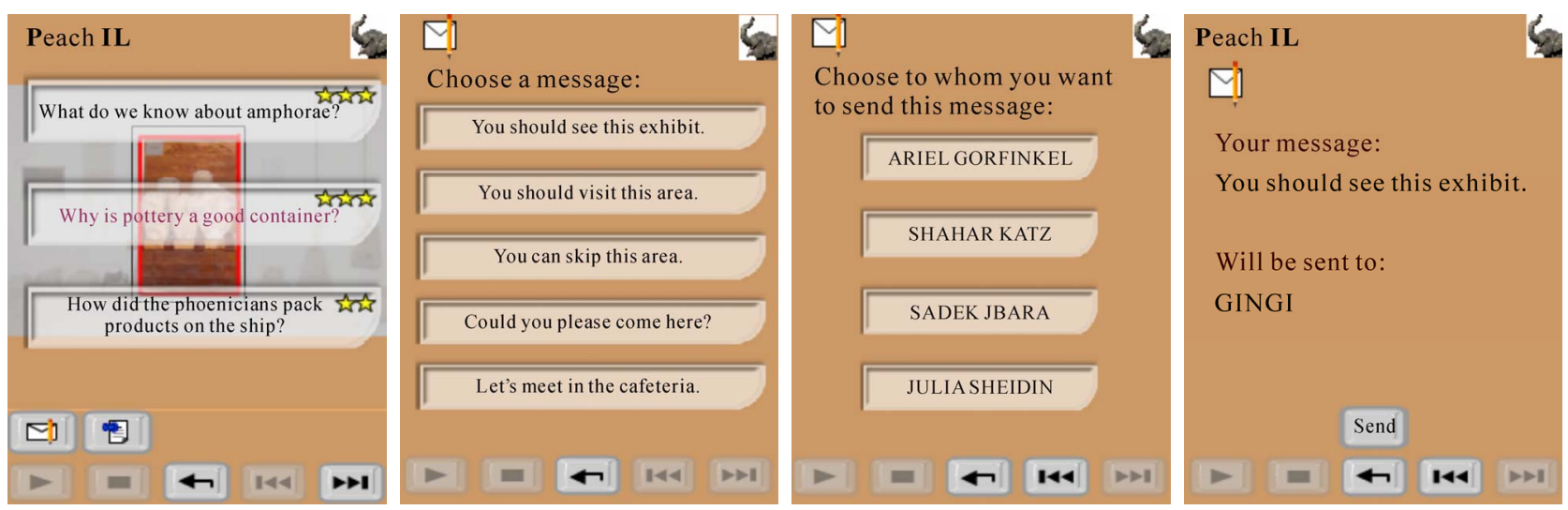

Figure 5. Sending a context aware immediate message.
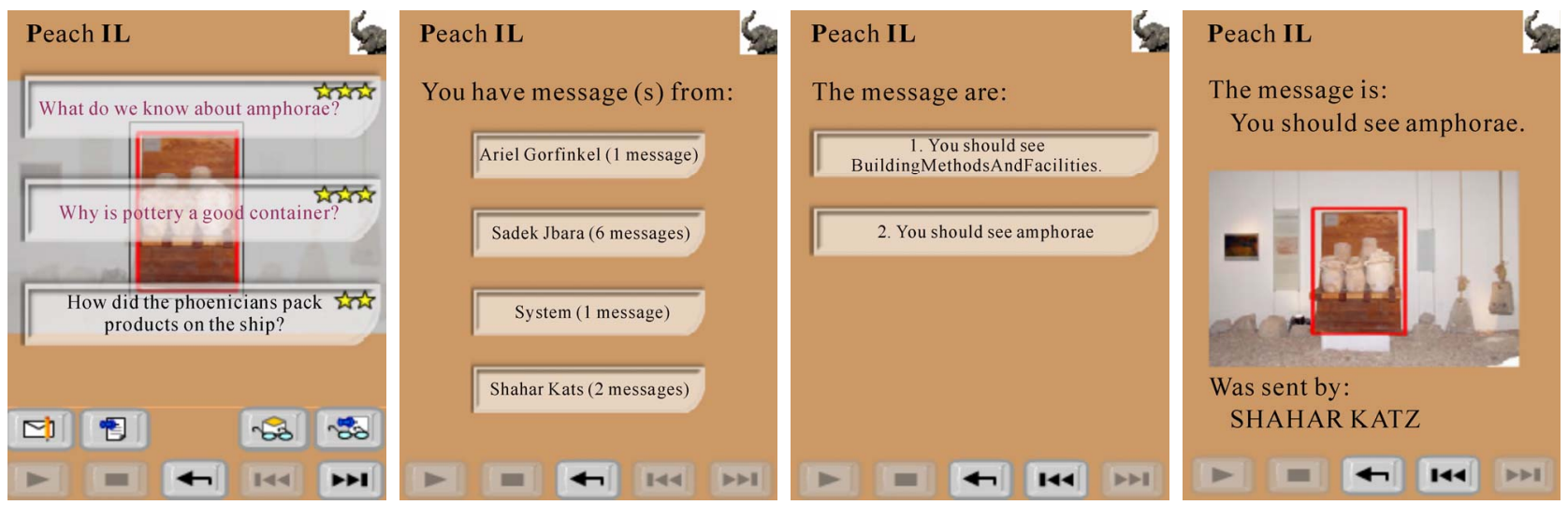

Figure 6. Receiving a context aware immediate message.

default values given to the variables $\mathrm{X}, \mathrm{Y}$, and $\mathrm{Z}$ in the $\mathrm{XML}$ configuration file $(\mathrm{X}=$ Presentation, $\mathrm{Y}=$ History [Presentation], $\mathrm{Z}=$ Exhibit).

\subsection{New Services Implemented as Proof-of Concept}

In addition to the above described SMS-like and Post-It services, two other services, Memories and Visit Reminder, were simulated using the generic framework, but not implemented as part of the visitor's guide system.

The Memories service adds contextual information about the environment to the visitor's annotations; the contextual information may refer to any type of object, such as exhibition, area, exhibit, and presentation. It enables the visitor to write comments in free text about objects of different types in the museum. This service is classified as a diary service.

The "Visit Reminder" service, which is also a diary service, provides a sequence of reminder services. Before the visit starts, the visitors may choose a list of locations that they plan to see during the visit within a specific time frame. The service has the goal to remind them "Don't miss" what they planned to see or listen to within the predefined time frame.

\subsection{Assessing the Generality of the Framework}

Above, we described four services which have been implemented or simulated on the basis of the proposed framework. This framework allows users to easily define and implement communication services in various applications, without the need to worry about the specific technical details, as demonstrated by the application described earlier. To assess our claim that this framework supports a wide range of communication services, we follow a descriptive evaluation method, called scenarios [41], which requires constructing detailed scenarios around the suggested artifact (the generic framework in our case) to demonstrate its utility.

In particular, Table 3 shows how the suggested framework supports all the communication services that were provided by the 12 systems reviewed in Section 2. The second column describes the communication services as provided by the different systems (Imm abbreviates immediate messages). Columns three to six describe how the same service can be defined using the generic framework and the last column comments on the status of the 
Table 3. Implementation of the various communication services using the generic framework.

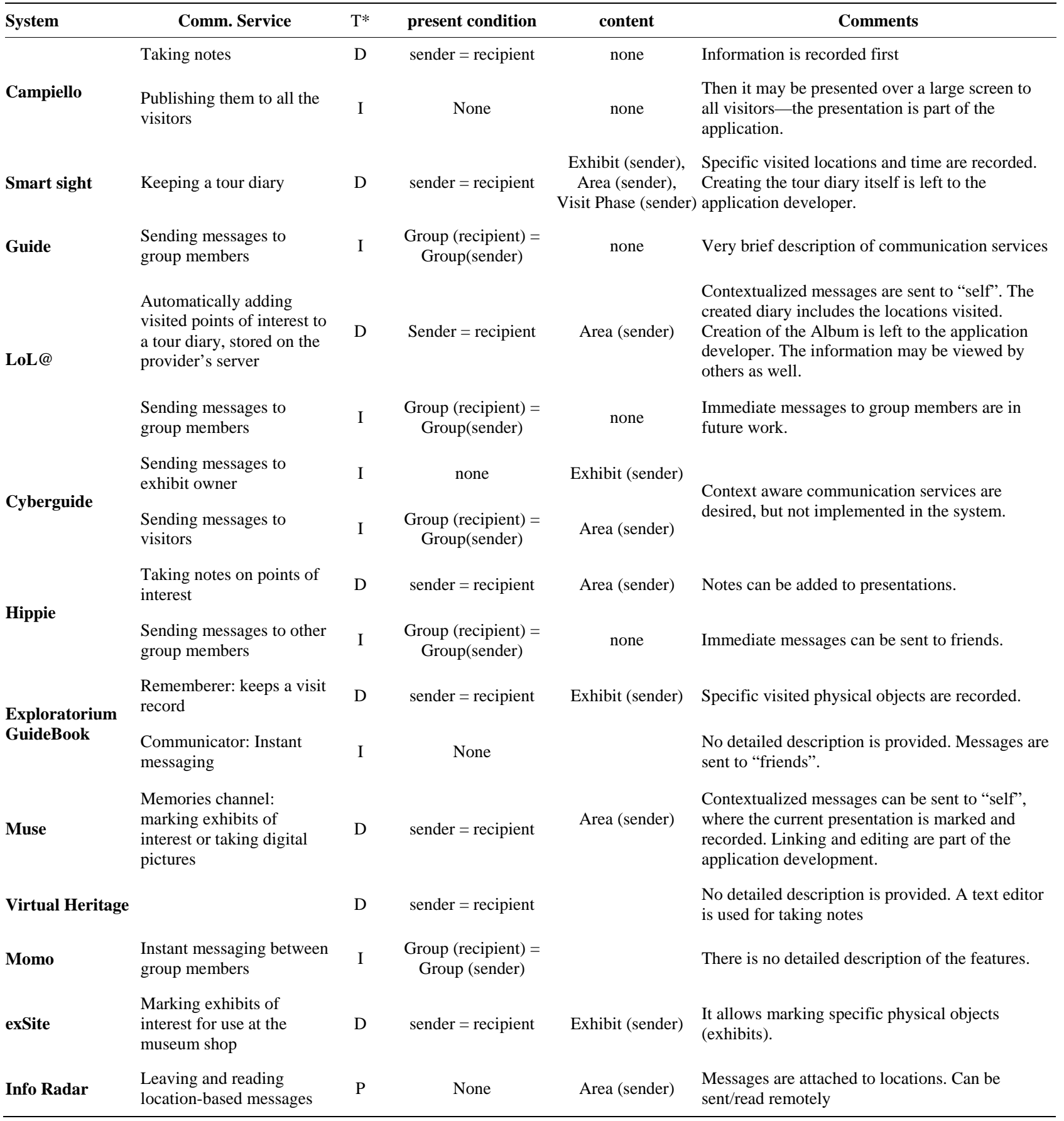

*D-diary, I-immediate, P-post it.

services' implementation and what remains for the application developer's responsibility. Empty cells mean that there was not enough information about the service to determine its contextual conditions or content.

Looking at the different rows of the table, we can see how every service in the reviewed systems can be implemented using the generic framework. The diary ser- vices, for example, namely those in Campiello, SmartSite, LOL@, HIPPIE, Exploratorium, GuideBook, MUSE, and exSite, are all characterized by a contextual present condition specifying that the sender equals to the recipient and no contextual future condition.

While the first characterization is essential to all diary services, the absence of contextual future condition is a 
matter of current reality: no system has yet implemented a diary service with post-it capabilities (e.g., a personal reminder service). Furthermore, all diary services in the reviewed systems partially support spatial context (in the form of area) and contextualized information (in the form of exhibits); they do not support temporal neither social context awareness.

Regarding immediate messages, one can see that the reviewed systems are at most social aware (referring to the sender's and recipient's groups) while delivering the messages. In addition, the messages themselves may contain spatial context (in the form of area) and contextualized information (in the form of exhibits). Our framework can provide more sophisticated immediate messaging (e.g., sending messages only to the visitors who have not yet been at the location of the sender are currently at a specific location). Finally, the post-it service of Info Radar refers to the location (area) of the recipient, with respect to the sender, and embeds spatial-related and contextualized information in the message to be sent. Again, while our generic framework supports these services, it is also capable of supporting more complicated post-it mechanisms.

While the basic context aware communication mechanisms are common, the applications themselves differ. As a result, a large variety of applications can be developed using the suggested framework. Note that the contextual conditions used in all the reviewed systems are quite simple and only partially use the attributes proposed by our framework.

Another dimension of the generality of our framework is its independence of the underlying infrastructure type. The configuration is expressed by means of an XML file, which makes it possible to use with different kinds of infrastructures over which these applications can be developed. Yet, it requires an interpreting component, as an interface with the infrastructure.

\section{Conclusions and Future Work}

Context aware communication services can play an important role in supporting visitors in cultural heritage sites, as demonstrated by the abundance of related work that exists. Our work shows that although there may be many "flavors" to communication services, they all share a small set of common characteristics. This calls for a standard definition of such services that can be applied in any application, so developers can define the specific "flavor" of services according to their system requirements, while reusing the common, generic infrastructure.

The framework suggested in this paper focuses on communication services. It defines two dimensions for classifying context aware communication services: the communication service type (namely immediate, post-it, or diary) and the supported context type (i.e., contextualized information, spatial context, temporal context, and social context). Given the abundance of context aware toolkits, it is possible to use any context management tool kit that can provide the required contextual data.

This framework has been implemented using two main components: a service configuration tool and a generic communication generator tool. The generic communication service relies on the LoudVoice multi-agent system. However, specific application components that tailor user interfaces to the communication services have to be developed separately for each communication service type. Since our conceptual framework is not dependent on a specific communication solution, future work will focus on developing a web service-based "Communication Generator" to allow applying the newly suggested framework to a widely used technology.

Although analyzed and evaluated in the context of Visitor's Guides, the suggested framework aims at facilitating the development of context-aware communication services in general, which nowadays is considered to be a complex and time-consuming task due to the lack of an adequate infrastructure support [42]. Furthermore, considering the increasing number of applications providing context-aware communication services to their users and the heterogeneity of communication services which an application may use, such a framework can be generalized for context-aware communication services in active environments in general. Furthermore, the contextual aspects can be extended to include, for instance, cognitive context of the senders and recipients, which includes their believed knowledge at a certain time. Indeed, this kind of context may partially be addressed in the current framework by relating to the visit history and assuming it represents what the visitor knows. In order to address a richer range of contextual aspects, developers may consider using a generic tool like the tool developed by [14] and others, and by doing so to focus on application specific aspects, rather than developing ad-hoc context aware communication services.

As for the future, we may use the framework presented here and apply it to context aware applications in general. Consider, for example, the area of context-aware shopping recommendations. Different applications can be developed in this domain, such as location-aware recommendation or recommendation of products that are supplementary to a product recently bought. These applications, as well as others, may share a common infrastructure. Similarly to our framework, a service configuration tool can be designed and implemented in that area. Such service configuration tool would build on a domain model which captures the unique features and relevant contextual attributes in that domain, and would streamline 
the development of such applications.

\section{Acknowledgements}

The research was supported by the collaboration project between the Caesarea-Rothschild Institute at the University of Haifa and FBK/irst in Trento and by FIRB project RBIN045PXH. Experimentation was conducted at the Hecht museum at the University of Haifa, Israel.

\section{REFERENCES}

[1] P. Marti, A. Rizzo, L. Petroni, G. Tozzi and M. Diligenti, "Adapting the Museum: a Non-Intrusive User Modeling Approach," Proceedings of the 7th International Conference on User Modeling, Banff, 20-24 June 1999. pp. 311-313.

[2] R. Oppermann and M. Specht, "A Context-Sensitive Nomadic Exhibition Guide," Proceedings of the 2nd Symposium on Handheld and Ubiquitous Computing, Bristol, 25-27 September 2000, pp. 31-54.

[3] A. Bright, J. Kay, D. Ler, K. Ngo, W. Niu and A. Nuguid, “Adaptively Recommending Museum Tours," Proceedings of Workshop on Smart Environments and Their Applications to Cultural Heritage, Tokyo, 11 September 2005, pp. 29-32.

[4] D. Petrelli and E. Not, "User-Centred Design of Flexible Hypermedia for a Mobile Guide: Reflections on the HyperAudio Experience," User Modeling and User-Adapted Interaction, Vol. 15, No. 3-4, 2005, pp. 303-338. doi:10.1007/s11257-005-8816-1

[5] L. Aroyo, N. Stash, Y. Wang, P. Gorgels and L. Rutledge, "Chip Demonstrator: Semantics-Driven Recommendations and Museum Tour Generation," Proceedings of the 6th International Semantic Web Conference, Busan, 11-15 November 2007, pp. 879-886.

[6] R. Damiano, C. Gena, V. Lombardo, F. Nunnari and A. Pizzo, "A Stroll with Carletto: Adaptation in DramaBased Tours with Virtual Characters," User Modeling and User-Adapted Interaction, Vol. 18, No. 5, 2008, pp. 417453. doi:10.1007/s11257-008-9053-1

[7] M. Rantanen, A. Oulasvirta, J. Blom, S. Tiitta and M. Mäntylä, "InfoRadar: Group and Public Messaging in the Mobile Context," Proceedings of the 3rd Nordic Conference on Human-Computer Interaction, Tampere, 23-24 October 2004, pp. 131-140.

[8] G. Leinhardt and K. Knutson, "Listening in on Museum Conversations,” AltaMira Press, Walnut Creek, 2004.

[9] J. Falk and L. Dierking, "The Museum Experience," Whalesback Books, Washington, 1992.

[10] S. Bitgood, "Environmental Psychology in Museums, Zoos, and Other Exhibition Centers,” In: R. Bechtel and A. Churchman Eds., Handbook of Environmental Psychology, John Wiley \& Sons, New York, 2002, pp. 461480.

[11] B. Gammon and A. Burch, "Designing Mobile Digital Experiences,” In: L. Tallon and K. Walker Eds., Digital
Technologies and the Museum Experience Handheld Guides and Other Media, AltaMira Press, Lanham, 2007, pp. 35-60.

[12] O. Stock and M. Zancanaro, "PEACH: Intelligent Interfaces for Museum Visits,” Cognitive Technologies Series, Springer, Heidelberg, 2007.

[13] T. Kuflik, O. Stock, M. Zancanaro, A. Gorfinkel, S. Jbara, J. Sheidin and N. Kashtan, "A Visitor's Guide in an Active Museum: Presentations, Communications, and Reflection," Association for Computing Machinery: Journal of Computers and Cultural Heritage, Vol. 3, No. 3, 2011.

[14] A. K. Dey, G. D. Abowd, "Towards a Better Understanding of Context and Context-Awareness," Proceedings of the 1st International Symposium on Handheld and Ubiquitous Computing, Karlsruhe, 27-29 September 1999, pp. 304-307

[15] L. Roffia, "Context Related Information Sharing and Retrieval in Mobile Cultural Heritage Applications,” Ph. D. Thesis, University of Bologna, Italy, 2004.

[16] R. Want, A. Hopper, V. Falcao and J. Gibbons, "The Active Badge location system," Association for Computing Machinery: Transactions on Information System, Vol. 10, No. 1, 1992, pp. 91-102. doi:10.1145/128756.128759

[17] N. Davies, K. Cheverst, K. Mitchell and A. Efrat, "Using and Determining Location in a Context-Sensitive Tour Guide,” Computer, Vol. 34, No. 8, 2001, pp. 35-41. doi:10.1109/2.940011

[18] J. Healey and R. W. Picard, "StartleCam: A Cybernetic Wearable Camera," Proceedings of the 2nd IEEE International Symposium on Wearable Computers, Pittsburgh, 19-20 October 1998, pp. 42-49.

[19] K. Henricksen, J. Indulska and A. Rakotonirainy, "Modelling Context Information Pervasive Computing Systems," Proceedings of the 1st International Conference on Pervasive Computing, Zurich, 26-28 August 2002, pp. 167-180.

[20] F. Siegemund, "A Context-Aware Communication Platform for Smart Objects,” Proceedings of the 2nd International Conference on Pervasive Computing, Linz, 18-23 April 2004, pp. 69-86.

[21] C. Anagnostopoulos, A. Tsounis, and S. Hadjiefthymiades, "Context Awareness in Mobile Computing Environments: A Survey,” Wireless Personal Communications, Vol. 42, No. 3, 2008, pp. 445-464.

[22] X. Zhang, J. Liao and J. Liu, "Open Middleware-Based Infrastructure for Context-Aware in Pervasive Computing," Proceedings of the 1st International Symposium on Computational and Information Science, Shanghai, 16-18 December 2004, pp. 85-92.

[23] A. Dey, T. Sohn, S. Streng and J. Kodama, "iCAP: Interactive Prototyping of Context-Aware Applications,” Proceedings of the 4th International Conference on Pervasive Computing, Dublin, 7-10 May 2006, pp. 254-271.

[24] Q.-S. He and S.-L. Tu, "A Lightweight Architecture to Support Context-Aware Ubiquitous Agent System," Proceedings of 9th Pacific Rim International Work- shop on Multi-Agents, Guilin, 7-8 August 2006, pp. 696- 701. 
[25] O. Stock, M. Zancanaro, P. Busetta, C. Callaway, A. Krüger, M. Kruppa, T. Kuflik, E. Not and C. Rocchi, "Adaptive, Intelligent Presentation of Information for the Museum Visitor in PEACH," User Modeling and UserAdapted Interaction, Vol. 17, No. 3, 2007, pp. 257-304. doi:10.1007/s11257-007-9029-6

[26] Z. Y. Yu, X. S. Zhou, Z. W. Yu, J. H. Park and J. H. Ma, "Imuseum: A Scalable Context-Aware Intelligent $\mathrm{Mu}$ seum System,” Computer Communications, Vol. 31, No. 18, 2008, pp. 4376-4382. doi:10.1016/j.comcom.2008.05.004

[27] A. Grasso, M. Koch and D. Snowdon, "Campiello-New User Interface Approaches for Community Networks," Association for Computing Machinery: SIGGROUP Bulletin, Vol. 20, No. 2, 1999, pp. 15-17.

[28] A. Agostini, G. De Michelis, M. Divitini, M. A. Grasso and D. Snowdon, "Design and Deployment of Community Systems: Reflections on the Campiello Experience," Interacting with Computers, Vol. 14, No. 6, 2002, pp. 689-712. doi:10.1016/S0953-5438(02)00016-4

[29] J. Yang, W. Yang, M. Denecke and A. Waibel, "Smart Sight: A Tourist Assistant System," Proceedings of the 3rd International Symposium on Wearable Computers, San Francisco, 18-19 October 1999, pp. 73-78.

[30] K. Cheverst, N. Davies, K. Mitchell and P. Smith, "Providing Tailored (Context-Aware) Information to City Visitors," Proceedings of the International Conference on Adaptive Hypermedia and Adaptive Web-based Systems, Trento, 28-30 August 2000, pp. 73-85.

[31] M. Umlauft, G. Pospischil, G. Niklfeld and E. Michlmayr, “LoL@, A Mobile Tourist Guide for UMTS," Journal of Information Technology and Tourism, Vol. 5, No. 3, 2003, pp. 151-164. doi:10.3727/109830503108751108

[32] S. Long, R. Kooper, G. D. Abowd and C. G. Atkeson, "Rapid Prototyping of Mobile Context-Aware Applications: the Cyberguide Case Study," Proceedings of the 2nd Annual International Conference on Mobile Computing and Networking, New York, 10-12 November 1996, pp. 97-107.

[33] R. Oppermann and M. Specht. "A Nomadic Information System for Adaptive Exhibition Guidance,” Archives and Museum Informatics, 1999, pp. 127-138.

$$
\text { doi:10.1023/A:1016619506241 }
$$

[34] G. Benelli, A. Bianchi, P. Marti, E. Not and D. Sennari, "HIPS: Hyper-Interaction within the Physical Space," Proceedings of IEEE International Conference on Multimedia Computing and Systems, Firenze, Vol. 2, 7-11 June 1999, pp. 1077-1078.

[35] M. Fleck, M. Frid, T. Kindberg, E. O’Brien-Strain, R. Rajani and M. Spasojevic, "From Informing to Remembering: Ubiquitous Systems in Interactive Museums," Pervasive Computing, Vol. 1, No. 2, 2002, pp. 13-21.

[36] F. Garzotto, T. Cinotti and M. Pigozzi, "Designing MultiChannel Web Frameworks for Cultural Tourism Applications: The MUSE Case Study," Archives and Museums Informatics, Toronto, 2003, pp. 239-354.

[37] E. Farella, D. Brunelli, L. Benini , B. Ricco and M. E. Bonfigli, "Pervasive Computing for Interactive Virtual Heritage,” IEEE MultiMedia, Vol. 12, No. 3, 2005, pp. 46-58. doi:10.1109/MMUL.2005.54

[38] J. Jaén, J. Mocholí, J. Esteve, V. Bosch and J. Canós, "MoMo: Enabling Social Multimedia Experiences in Hybrid Museums," Proceedings of International Workshop of Re-Thinking Technology in Museums: Towards a New Understanding of People's Experience in Museums, Limerick, 29-30 June 2005, pp. 245 -251.

[39] P. Busetta, A. Doná, and M. Nori, "Channeled Multicast for Group Communications,” Proceedings of the 1st International Joint Conference on Autonomous Agents and Multi-Agent Systems, Bologna, 15-19 July 2002, pp. 1280-1287.

[40] P. Busetta, T. Kuflik, M. Merzi and S. Rossi, "Service Delivery in Smart Environments by Implicit Organizations," Proceedings of the 1st Annual International Conference on Mobile and Ubiquitous Systems: Networking and Services, Boston, 22-26 August 2004, pp. 356-363.

[41] A. R. Hevner, S. T. March and J. Park, "Design Science in Information Systems Research,” MIS Quarterly, Vol. 28, No. 1, 2004, pp. 75-105.

[42] G. Chen and D. Kotz, "A Survey of Context-Aware Mobile Computing Research,” Technical Report TR2000-381, Department of Computer Science, Dartmouth College, Hanover, 2000. 